\title{
Estimation of Optimum Rendezvous Point for Mobile Sink (ORP-MS) in WSN
}

\author{
Vrince Vimal ${ }^{1}$, Madhav J Nigam ${ }^{2}$ \\ Department of Electronics and Communication Engineering, Indian Institute of Technology, Roorkee, India \\ *Corresponding Author E-mail: ${ }^{1}$ vrince.vimal@gmail.com, ${ }^{2}$ mkndnfec@iitr.ac.in
}

\begin{abstract}
Clustering of the sensors in wireless sensor network is done to achieve energy efficiency. The nodes, which are unable to join any cluster, are referred to as isolated nodes and tend to transfer information straight to the base station. It is palpable that isolated nodes and cluster heads communicate with the base station and tend to exhaust their energy leaving behind coverage holes. In this paper, we propose the innovative clustering scheme using mobile sink approach to extend networks lifetime. The proposed (ORP-MS) algorithm is implemented in MATLAB 2017a and the results revealed that the proposed algorithm outdid the existing algorithms in terms networks lifetime and energy efficiency simultaneously achieved high throughput.
\end{abstract}

Keywords—WSN; Clustering protocol; Mobile Sink; Tour Plan; Energy efficiency; Network lifetime.

\section{Introduction}

Wireless Sensor Networks (WSN) are jam-packed with ambiguity and inaccuracies are prevalent to it. Previous years have seen lot of research in field of WSN. Though, challenges and probabilistic processes are innate to WSN, yet it finds vast application in industrial as well as military applications[1]-[3].

WSN integrate vast measure of elfin sensor nodes which consist of a radio interface, processor, A to D converter, sensors, memory, and a battery. Nodes are required to sense, collect, save and propel data to sink whenever required using radio communication [4]. In Random, deployment nodes are usually scattered (Fig-1). The density of nodes can be controlled but up to some extent [5].

This technique is best suited for the bids where Region of observation (ROI) is unapproachable e.g. war zones.

Clustering is done to achieve energy efficiency in arbitrarily deployed sensor network [6]. In Clustering scheme group of nodes form a cluster and elect one node as Cluster Head (CH), which is responsible to communicate with the base station (BS). Information sensed by the sensors is sent to $\mathrm{CH}$, which temporarily stores it.

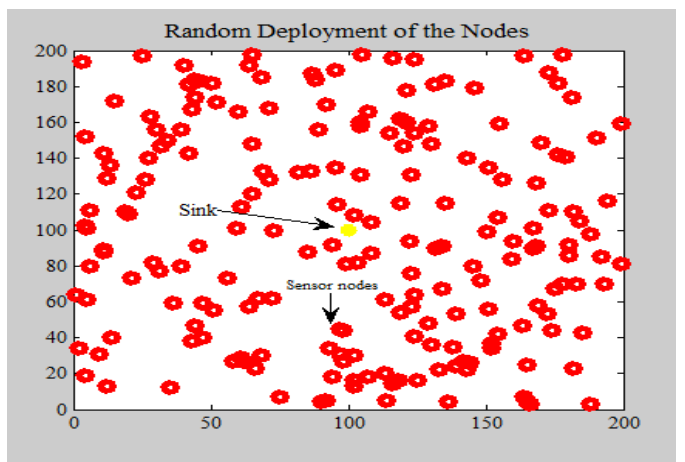

Fig. 1: Random Deployment

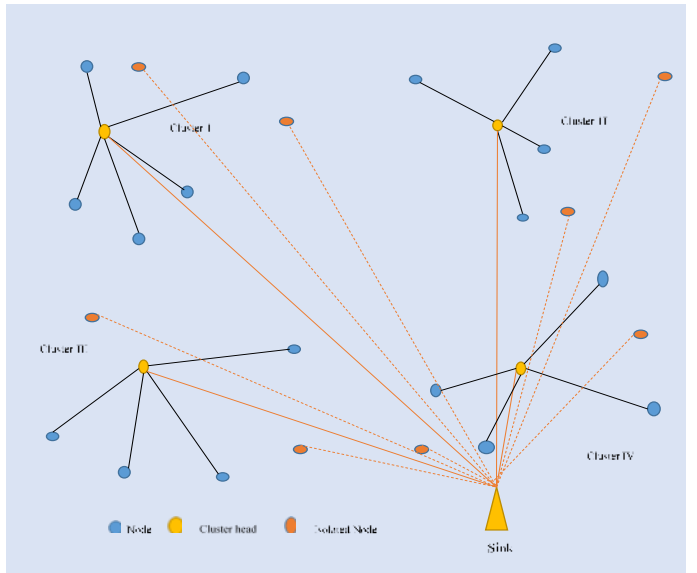

Fig. 2: Clustered WSN with Remote Sink

Finally the information gathered by nodes is communicated to BS, after fixed duration of time [3], [7]-[11]. In clustering the nodes which are unable to join the cluster are called as isolated nodes and are responsible for communicating information directly to the Base station (BS) [12]. Now as is apparent that either Cluster Head $(\mathrm{CH})$ or the isolated node communicates with the $\mathrm{BS}$, so, $\mathrm{CH}$ and isolated node tend to drain their energy soon in comparison with other sensors deployed in same network. The dead nodes give rise to coverage holes known as coronas in the network[13].

In the stationary sink setup of WSN the nodes which are situated nearer to the sink are extra prone to drain their energy before other nodes in the network, due to concentration of gen towards sink.

The movement of gen in various application follow many to one protocol where the nodes near to the sink heavy traffic as compared to other nodes. Therefore, sensors near to the sink diminish their energy sooner, causing formation of coronas. This snag is addressed effectively in[12], [14]. These dead nodes cause ailment in the network and reduces the sensing reportage of the network. 
Additionally, with more dead nodes around the sink, the sink might become unreachable and data collected by modes may not be retrieved.

Subsequently, algorithms for stationary base station to conform load matching has to mitigate uniform energy consumption in the system. This is why the mobile sink came to picture [15]-[17].

In this study, we propose an innovative algorithm, using the model of mobile sink, which plummets the energy consumption of $\mathrm{CH}$ and isolated nodes during communication with sink. Entire geographic area to be observed by the network is divided in virtual zones. The localization[18] algorithm is implemented so that each nodes become aware of its coordinates. Then, clustering is implemented leading every zone to have its cluster and cluster head. Every zone sends information regarding location, cluster head ID, and cluster id to the base station. This information enables BS to identify isolated nodes in every zone.

Depending on the information gathered regarding isolated nodes, BS now identify the location in each zone to move for data collection. The location is fixed such that the coordinates of the rendezvous point is approximately in between cluster head and isolated nodes, which tentatively is the region to develop the coronas in future and helps prolonging the appearance of coronas in the network. Hence, prolongs the networks lifetime.

This paper is further organized as follows. Section II gives the background of the work carried out until date, section III defines the system model having energy saving analysis of proposed scheme and there after results are discussed followed by concluding statement.

\section{Related Work}

Non-uniform energy diminution is a bodacious issue of the clustering protocols with immovable sink for WSNs. Cluster heads and isolated nodes invest extra energy to communicate unswervingly with the base station. This causes non-uniform energy consumption in the network resulting in uncovered regions plummeting efficiency of the network. Energy conservation is a vital snag in WSN and is dually addressed by investigators all around the globe. Energy efficient clustering schemes having stationary base station are presented in[3], [7], [11], [19]. Mobile Sink schemes are elaborated in[16], [17], [20]-[29]. Mobile sinks are gathering a lot of attention these days, lot of researchers have investigated the option of mobile sink in smart city network as well. To resolve energy conservation issue for WSN, the authors in [22] suggest a Distributed Energy-efficient Clustering Algorithm for moving-sink centered WSNs, where the sink travels about the target zone with a static route and velocity. The suggested clustering procedure, binds individual sensor node with a $\mathrm{CH}$ by means of single or multi-hop communiqué, where a $\mathrm{CH}$ conveys its information packet to the sink once distance among them is least. Consequently, the precise movement of the sink round the network aids in matching the energy depletion of the sensor networks. The investigational outcomes prove the competence of their suggested procedure over the present state-of-the-art procedures in terms of dissimilar metrics like, network lifespan, energy depletion, etc.

A Virtual Grid based Dynamic Routes Adjustment (VGDRA) system for the mobile sink was presented in[24] to curtail the cost of communication. Authors divided the entire geographic region to smaller areas named grids and the nodes, which were close to center, were elected as advance nodes. Cell header nodes concept was built. The sink traversed the geographic region collecting data from header nodes. Authors in[25] suggested mixed integer programming and extended the lifetime using mobile sink. In[26] convex optimization model was built using support vector regression to suggest ideal trajectory for sink, which in turn effected the networks lifespan. The Wireless Charging Vehicle was used in [29], which was used to traverse the network area and charge the sensor nodes without any physical contact.

$\operatorname{In}[28]$, the authors deliberated the routing algorithm which rotates around on the optimal number of hops amid source and sink. The classical use of mixed-integer programming based on the
Lagrangian relaxation scheme, to pronounce crucial parameter, which oversee the hop-by-hop switching. Author in [27] presented a safe clustering algorithm for mobile sink scenario. The approach presented was to keep a watch on malicious node and remove it as soon as it is detected. They proposed minimum and well-balanced clusters for WSN.

The author in [30] proposed DCFly i.e. data collection fly which was well suited for random deployment in harsh terrain. Miniature aerial vehicle was proposed to collect data by flying above the deployed field. Author in [31] proposed multi sink placement in wireless sensor networks.

All the above work based on cluster and non-clustered structure with mobile sink have either not taken into account the presence of isolated nodes, while deciding the rendezvous point for the sink node or they have considered the continuous movement of the sink around the network during the data movement tour of the sink. Models with the continuous movement of the sink node would give less communication time with the cluster heads which furthermore leads to lesser throughput of the network. On the other hand, the models sans the presence of the isolated nodes would lead to more energy consumption by the isolated nodes as the distance of the sink from the isolated would be more.

\section{System model}

\section{A. Motivation}

Agility, Detecting and interaction with the base station, are the processes, which requires energy of sensor node. After clustering algorithm is implemented, some nodes become isolated due to various reasons. If isolated node is at far off from base station, isolated node is destined to drain its energy causing incomplete coverage thereby reducing networks lifetime. If second stage of clustering is done for isolated nodes [3] and mobile sink route is planned such that the rendezvous point is selected to be a centroid of header nodes which finally transfer data to sink networks life time can be overwhelmingly augmented.

\section{B. Network Model}

Let the entire network of area $N \times N$ square units be alienated in to ' $M$ ' zones with area of $K \times K$ square units. Also presume that 'nod ${ }_{i}$ ' be the number of nodes in ' $i$ 'th ' zone of the network. Suppose ' $\rho_{s}$ ' is the density of nodes in zone ' $i$ ', of area $K \times K$ square units. (For the sake of simplicity and understanding, we assume network to be divided into 4 zones)

\section{Notations}

The following notations are used in this paper:

1) $n=\left\{n_{1}, n_{2} \ldots n_{i}\right\}$, set of sensor nodes, where $n_{i}$ is individual node.

2) $\mathrm{CH}=\left\{\mathrm{CH}_{1}, \mathrm{CH}_{2} \ldots \mathrm{CH}_{i}\right\}$, set of Cluster Heads, where $\mathrm{CH}_{i}$ is individual $\mathrm{CH}$.

3) $R p=\left\{R p_{1}, R p_{2}, \ldots . . R p_{m}\right\}$, Rendezvous points in each zone

\section{Elucidation}

1) Cluster Head. The advance node elected to transmit information of sensors in cluster to the sink node.

2) Connected node. The nodes which are part of cluster and sends its data to sink node via $\mathrm{CH}$.(con)

3) Isolated nodes. The nodes, which are not in communicating range of $\mathrm{CH}$ and sends its data directly to sink node. (uncon)

4) Zone. The part of network having its own Cluster Head 


\section{E. Localization Model}

In the proposed study, initially, Received Signal Strength Indicator (RSSI) based adaptive localization algorithm is used to approximate the coordinates of each node. We place 3 lantern nodes for enabling rest of the nodes to evaluate their co-ordinates. We make one more assumption that path loss exponent for the pair of nodes will remain same even if their relative positions ae shifted. For sake ofunderstanding, we take an example of node's' in Fig-4 below. Node is at distance of $d_{l a}$ from lantern node $l_{a}$ at $\left(x_{l a}, y_{l a}\right), d_{l b}$ from lantern node $l_{b}$ at $\left(x_{l b}, y_{l b}\right)$ and at $d_{l c}$ from $l_{c} a t\left(x_{l c}\right.$, $y_{l c}$ ). Some symbols used in this study are listed in Table I.

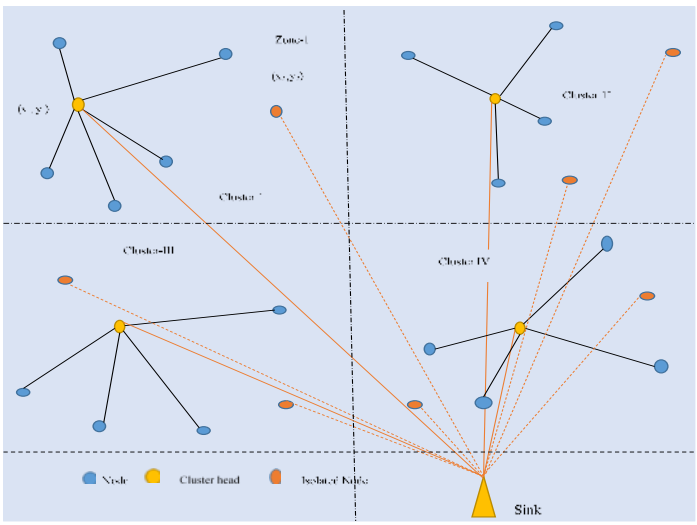

Fig. 3: Network Partitioned in 4 zones

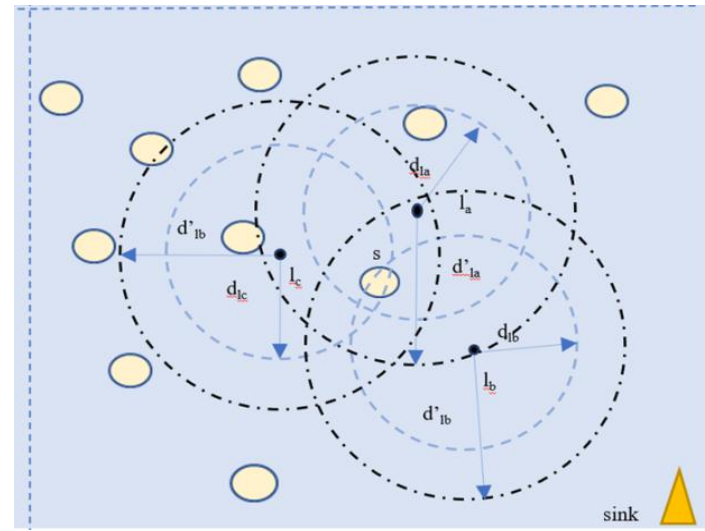

Fig. 4: Localization

Table 1: Symbols

\begin{tabular}{|c|c|}
\hline$M$ & Number of zones in network \\
\hline$N \times N$ & Network area \\
\hline$K \times K$ & Area of the individual zone \\
\hline$\rho_{s}$ & Density of nodes in zone \\
\hline nod $_{i}$ & Number of nodes in $i^{\text {th }}$ zone \\
\hline $\mathrm{CH}_{\mathrm{i}}$ & Cluster head of $i^{\text {th }}$ zone \\
\hline$\mu$ & Number of nodes in cluster \\
\hline$\beta$ & Number of isolated nodes \\
\hline$a$ & Area covered by nodes in cluster \\
\hline$b$ & Area covered by isolated nodes \\
\hline$r$ & Corona prone area \\
\hline$P$ & Packet size \\
\hline$E_{\text {elec }}$ & Energy consumed in transmitter \\
\hline$E_{a m p}$ & Energy consumed in transmitter amplifier \\
\hline$d_{i}$ & Distance of $i^{\text {th }}$ node from cluster head \\
\hline$d_{i}^{\prime}$ & Distance of $i^{\text {th }}$ isolated node from cluster head of isolated node \\
\hline$d_{I S O}$ & Distance of isolated node from base station (Sink) \\
\hline$d_{I S O}^{\prime}$ & Distance of $\mathrm{CH}$ of isolated node from base station (Sink) \\
\hline$r_{\text {ela }}$ & Relative error \\
\hline$r_{v}$ & RSSI value of node \\
\hline
\end{tabular}

Assuming that the predicted value of distance of nodes from lantern is predicted with error. Let $d^{\prime}{ }_{l a},{ }^{\prime}{ }_{l b}$ and $d^{\prime}{ }_{l c}$ be the predicted distances.

So the coordinates of node $\mathrm{s}\left(\mathrm{x}_{\mathrm{s}}, \mathrm{y}_{\mathrm{s}}\right)$ can be estimated as -

$$
\begin{aligned}
& \sqrt{\left(\left(x_{s}-x_{l a}\right)^{2}+\left(y_{s}-y_{l a}\right)^{2}\right)} \leq d_{l a}^{\prime} \\
& \sqrt{\left(\left(x_{s}-x_{l b}\right)^{2}+\left(y_{s}-y_{l b}\right)^{2}\right)} \leq d_{l b}^{\prime} \\
& \sqrt{\left(\left(x_{s}-x_{l c}\right)^{2}+\left(y_{s}-y_{l c}\right)^{2}\right)} \leq d_{l c}^{\prime}
\end{aligned}
$$

Now replace node ' $\mathrm{s}$ ' by the lantern nodes ' $l \mathrm{a}$ ' and estimating its coordinates $\left(x_{l a}^{\prime}, y_{l a}^{\prime}\right)$ by Eq. 1 . Similarly obtaining $\left(x_{l b}^{\prime}, y_{l b}^{\prime}\right)$ and $\left(x_{l c}^{\prime}, y_{l c}^{\prime}\right)$. Now relative error in desired measurement can be calculated as -

$r_{\text {ela }}=\frac{\sqrt{\left(\left(x_{l a}-x_{l a}^{\prime}\right)^{2}+\left(y_{l a}-y_{l a}^{\prime}\right)^{2}\right)}}{d_{l a}}$

Where, $d_{l a}=\sqrt{\left(\left(x_{l a}-x_{s}\right)^{2}+\left(y_{l a}-y_{s}\right)^{2}\right)}$

Hence, relative error in node position can be obtained. Once the coordinates of nodes are froze, it now act as lantern to enable other nodes to evaluate their positions and subsequently the position of all the sensor nodes in the network is obtained

\section{F. Mobile Sink}

In proposed algorithm, sink moves to every zone at rendezvous point for data collection. After localization, coordinates of all the nodes in all the zones are known. Rendezvous point is selected such that it is equidistant from $\mathrm{CH}$ and isolated nodes as shown in fig. 8. Sink pause for predefined duration at rendezvous point and then moves to next rendezvous point in next zone.

\section{G. Clustering}

In proposed scheme, the residual energy and ratio of distance from the center of the zone are taken into account to elect Cluster heads[3]. CH sends ping to rest of the nodes to join cluster. Once the clustering is done, isolated nodes are selected.

Now isolated nodes check whether they are in communicating range with any other isolated nodes, if yes, then they form a cluster of isolated nodes and elect $\mathrm{CH}$ of isolated node on basis of residual energy.

Since the nodes are randomly scattered in the network, this prompts to define the hypothesis that each zone will have nonuniform node density. For the zone of $K \times K$ sq. Units with 'nod number of nodes, density of nodes can be inferred as

$\rho_{s}=\frac{\operatorname{nod}_{i}}{K \times K}$

Now assuming that out of ' od $_{i}$ ' number of nodes, ' $\mu$ ' number of nodes have joined cluster and ' $\beta$ ' number of nodes are left isolated. Now density can be rearticulated as,

$\rho_{s}=\frac{\mu+\beta}{K \times K}$.

Supposing in zone ' $i$ ', ' $a$ ' Sq. units is the area enclosed by nodes in cluster and ' $b$ ' is the area enclosed by isolated nodes such that

$$
K \times K=a+b+r \text { sq.units }
$$

Where ' $r$ ' is the corona prone region as shown in Fig. 5

Now all the nodes in cluster that is in area ' $a$ ' will be transmitting data packets to $\mathrm{CH}$. So, energy spent in this radio transmission, 


$$
E_{1}=\left\{\sum_{i=1}^{\mu}\left(p \times E_{\text {elec }}+p \times E_{\text {amp }} \times d_{i}^{2}\right)\right\},
$$

where ' $d_{i}$ ' is the distance of ' $i$ ' ' node from $\mathrm{CH}$.

Now all the isolated nodes that is in area ' $b$ ' will be transmitting data packets directly to sink. So, energy spent in this radio transmission

$$
E_{2}=\left\{\sum_{i=1}^{\beta}\left(p \times E_{\text {elec }}+p \times E_{\text {amp }} \times d_{\text {ISO }}^{2}\right)\right\} .
$$

Cluster head will be transmitting received packets from nodes to sink, energy spent by $\mathrm{CH}$ for transmitting packets to base station,

$$
E_{3}=\left(p \times E_{\text {elec }}+p \times E_{\text {amp }} \times d_{C H}^{2}\right),
$$

So, the total energy spent by nodes in zone 1 for 1 transmission cycle

$$
E=E_{1}+E_{2}+E_{3} .
$$

According to proposed the sink should move to rendezvous point for collection of information.

Rendezvous point is selected such that it lies at equal distance from $\mathrm{CH}$ of isolated nodes, left over nodes and $\mathrm{CH}$ as shown in fig. 7 and can be evaluated as:

$$
R p=\left\{\frac{x_{c h}+\sum_{i=1}^{\beta} x_{I S O}}{\beta+1} ; \frac{y_{c h}+\sum_{i=1}^{\beta} y_{I S O}}{\beta+1}\right\},
$$

Where, $\beta$ is left over isolated nodes.

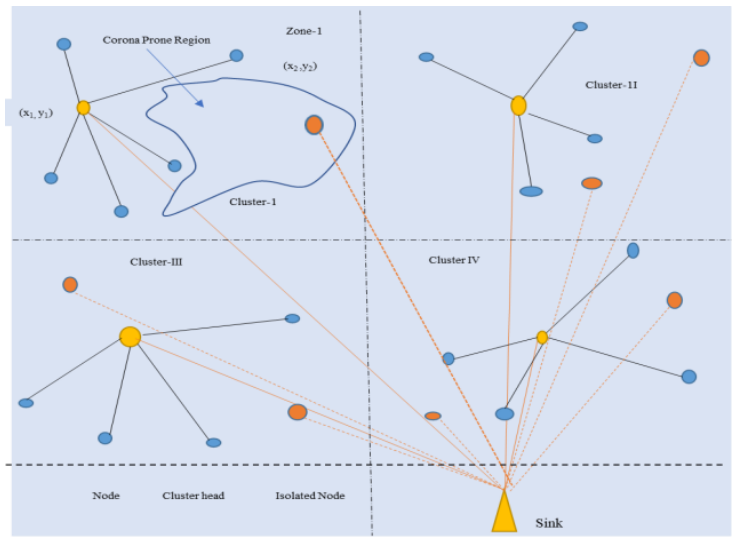

Fig 5: Clustering with Corona Prone Region

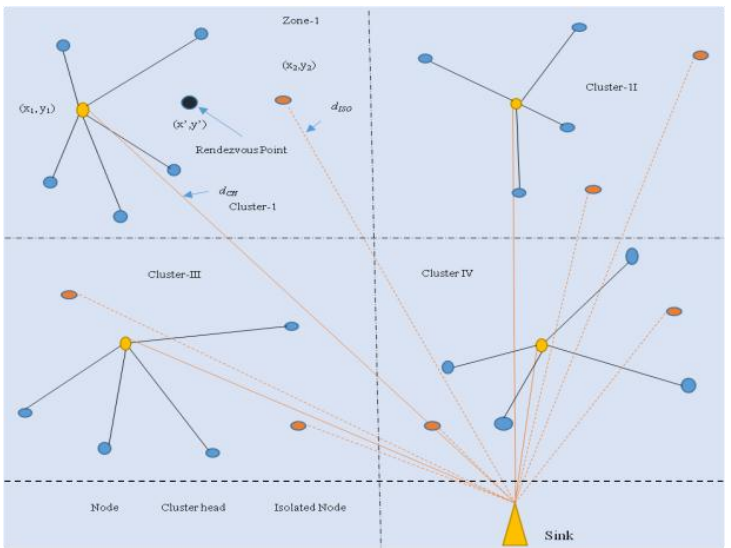

Fig. 6: Rendezvous Point
Considering the scenario, we have (i) nodes which transmit its data to $\mathrm{CH}$, (ii) Cluster head, (iii) Cluster head of isolated nodes and (iv) Nodes, which were not in communication range of any another node.

Energy spent by the nodes to transfer information packets to the $\mathrm{CH}$ shall be the same as $E_{l}$ 'i.e.

$$
E_{1}^{\prime}=E_{1}=\left\{\sum_{i=1}^{\mu}\left(p \times E_{\text {elec }}+p \times E_{\text {amp }} \times d_{i}^{2}\right)\right\},
$$

Assuming that after clustering of isolated nodes $\alpha$ node still remain isolated. These nodes will transmit their data packets directly to the sink. Energy exhausted by these nodes:

$$
E_{2}^{\prime \prime \prime}=\left\{\sum_{i=1}^{\alpha}\left(p \times E_{\text {elec }}+p \times E_{\text {amp }} \times\left(d_{i}^{\prime \prime}\right)^{2}\right)\right\},
$$

where, ' $d$ ' ' is the distance of isolated node from sink.

To calculate the energy drained by isolated nodes in cluster, energy spent by isolated nodes to transfer data to $\mathrm{CH}$ of isolated nodes:

$E_{2}^{\prime \prime}=\left\{\sum_{i=1}^{\beta-\alpha-1}\left(p \times E_{\text {elec }}+p \times E_{\text {amp }} \times\left(d_{i}^{\prime}\right)^{2}\right)\right\}$,

where ' $d_{i}$ ' is distance of ' $i$ th' isolated node from $\mathrm{CH}$ of isolated node.

Energy exhausted by $\mathrm{CH}$ of isolated node to transfer data packets to sink can be calculated as:

$$
E_{2}^{\prime}=\left(p \times E_{\text {elec }}+p \times E_{\text {amp }} \times\left(d_{\text {ISO }}^{\prime}\right)^{2}\right),
$$

Finally, the energy drained by $\mathrm{CH}$ in proposed scheme to transfer information to mobile sink,

$$
E_{3}^{\prime}=\left(p \times E_{\text {elec }}+p \times E_{\text {amp }} \times\left(d_{C H R}^{\prime}\right)^{2}\right),
$$

Now the total energy saved

$$
\begin{aligned}
& E_{\text {saved }}=E_{1}+E_{2}+E_{3}-E_{1}^{\prime}-\left(E_{2}^{\prime}+E_{2}^{\prime \prime}+E_{2}^{\prime \prime \prime}\right)-E_{3}^{\prime}, \\
& E_{\text {saved }}=E_{1}-E_{1}^{\prime}+E_{2}-\left(E_{2}^{\prime}+E_{2}^{\prime \prime}+E_{2}^{\prime \prime \prime}\right)+E_{3}-E_{3}^{\prime}
\end{aligned}
$$

From equation 12 we have, $E_{l}=E_{l}^{\prime}$. So, equation 17 reduces to

$$
E_{\text {saved }}=E_{2}-\left(E_{2}^{\prime}+E_{2}^{\prime \prime}+E_{2}^{\prime \prime \prime}\right)+E_{3}-E_{3}^{\prime}
$$

Putting the values of $E_{2}, E_{3}$ and $E_{2}^{\prime}, E^{\prime \prime}{ }_{2}, E_{3}{ }_{3}$

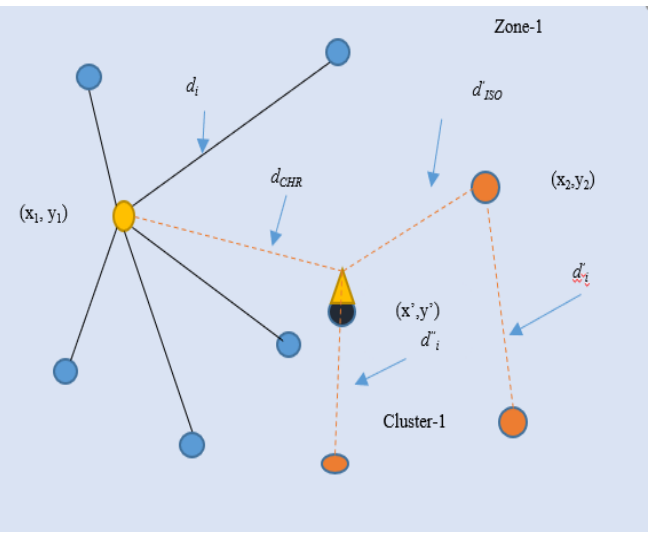

Fig. 7: Proposed scenario for zone-1 Isolated Node 


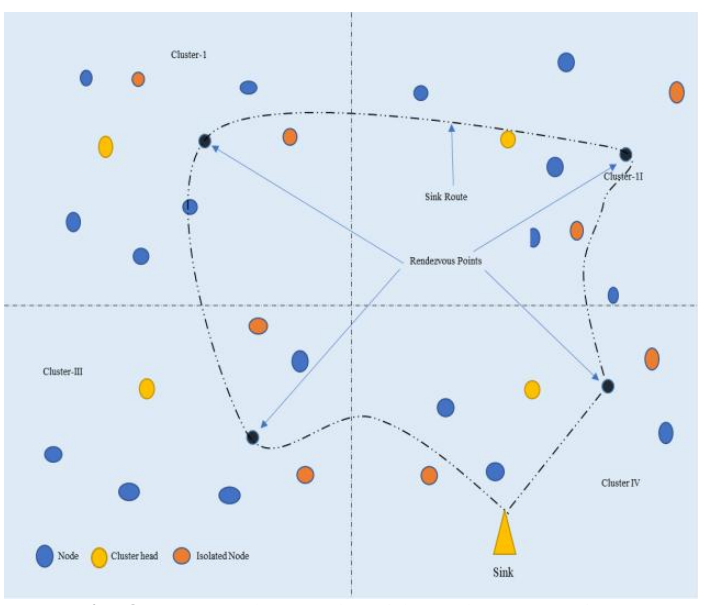

Fig. 8: Proposed scenario with rendezvous points

$E_{\text {saved }}=\left\{\sum_{i=1}^{\beta}\left(p \times E_{\text {elec }}+p \times E_{\text {amp }} \times d_{\text {ISO }}^{2}\right)\right\}-\left\{\begin{array}{l}\left(p \times E_{\text {elec }}+p \times E_{\text {amp }} \times\left(d_{\text {ISO }}^{\prime}\right)^{2}\right)+ \\ \sum_{i=1}^{\beta-\alpha-1}\left(p \times E_{\text {elec }}+p \times E_{\text {amp }} \times\left(d_{i}^{\prime}\right)^{2}\right)+ \\ \sum_{i=1}^{\alpha}\left(p \times E_{\text {elec }}+p \times E_{\text {amp }} \times\left(d_{i \prime}^{\prime \prime}\right)^{2}\right)\end{array}\right\}$

$+\left(p \times E_{\text {elec }}+p \times E_{\text {amp }} \times d_{C H}^{2}\right)-\left(p \times E_{\text {elec }}+p \times E_{\text {amp }} \times\left(d_{\text {CHR }}^{\prime}\right)^{2}\right)$

Assuming, $d_{I S O} \sim d^{\prime} \sim d^{\prime}{ }_{i}=d_{I S O}$, for sake of simplicity in analysis, we have,

$E_{\text {saned }}=\left\{\sum_{i=1}^{\beta}\left(p \times E_{\text {elec }}+p \times E_{\text {anp }} \times d_{\text {ISO }}^{2}\right)\right\}-\sum_{i=1}^{\beta}\left(p \times E_{\text {elec }}+p \times E_{\text {anp }} \times\left(d_{\text {ISO }}^{\prime}\right)^{2}\right)+p \times E_{\text {amp }}\left(d_{C H}^{2}-d^{2}{ }_{C H R}\right.$

$E_{\text {saved }}=p \times E_{\text {amp }} \times\left\{\sum_{i=1}^{\beta}\left(d_{\text {ISO }}^{2}-d^{\prime 2}{ }_{I S O}\right)+\left(d_{C H}^{2}-d_{C H R}^{\prime 2}\right)\right\}$

It is obvious that $\left(d_{I S O} \square d_{I S O}^{\prime}\right) \&\left(d_{C H} \square d_{C H R}^{\prime}\right)$

Therefore, $E_{\text {saved }} \cong p \times E_{\text {amp }} \times\left\{\sum_{i=1}^{\beta}\left(d_{I S O}^{2}\right)+\left(d_{C H}^{2}\right)\right\}$

For every rotund new cluster head is designated, isolated nodes are recognized, and contingent on this information, new location for mobile BS is designated. Base Station moves from zone to zone for collecting data from rendezvous point (Fig.8) reducing energy consumption of the Cluster Head and Isolated nodes.

The duration for which mobile base station stays in every zone is predefined to make communication between nodes moreeffective. This entire algorithm saves the energy of the nodes, which have highest probability to drain their energy and create coronas in the network. Hence, networks life is augmented.

\section{H. Algorithm for clustering in ORP-MS}

\author{
for $\mathrm{j}=1: \mathrm{M}$ \\ fori $=1: \operatorname{nod}_{i}$ \\ if R.E. $\left(\operatorname{nod}_{\mathrm{i}}\right)==$ highest \\ set status as $\mathrm{CH}$ \\ else \\ set status Cluster member$$
\text { end }
$$ \\ end \\ end \\ for each $\mathrm{CH}_{\mathrm{i}} \in \mathrm{CH}$ do \\ $\mathrm{CH}_{\mathrm{i}}$ broadcast ADV message to its neighbors \\ end \\ for each $\operatorname{nod}_{k} \in \mathrm{Ndo}$ \\ if $\mathrm{Adv}$. message = received from $\mathrm{CH}_{\mathrm{i}} \in \mathrm{CH}$ \\ $\operatorname{nod}_{k}$ becomes part of the connected set
}

CoNSet $\left[\right.$ con] $=\operatorname{nod}_{\mathrm{k}}$

con++

else

nod $_{\mathrm{k}}$ becomes part of unconnected set

UnCoNSet [uncon] $=\operatorname{nod}_{\mathrm{k}}$

uncon++

end

end

for each $\operatorname{nod}_{k}$ do

ifnod $_{\mathrm{k}} \in$ ConSet

Compute RSSI $->\mathrm{CH}_{4}$

RSSI val[rv]=RSSI - > $\mathrm{CH}_{\mathrm{i}}$

rv++

end

Cluster $[\mu]=\operatorname{Min}\{$ RSSI val[rv] $\}$

$\mu++$

if Sensor node $\in$ UnConSet

if $\operatorname{Dist}(\operatorname{nod}(\mathrm{k})->\operatorname{nod}(\mathrm{k}+1))<\mathrm{R}$

send ISO_Join message to form cluster

end

end

ifISO_Join message $=$ received

Update CoNSet: CoNSet $[$ con $]=\operatorname{nod}_{k}$

Con++

Update UnCoNSet: UnCoNSet[uncon]- $\operatorname{nod}_{k}$

uncon--

else

$\operatorname{IsoSet}[\beta]=\operatorname{nod}_{k}$

$\beta++$

end

end

Compute Rendezvous points in each zone

$\mathrm{Rp}=\left\{\mathrm{Rp}_{1}, \mathrm{Rp}_{2}, \ldots . . \mathrm{Rp_{ \textrm {m } }}\right\}$

$\mathrm{CH}$ Distribute TDMA schedule to the cluster members

Start data collection tour

for each point in $\mathrm{Rp}$

Move to rendezvous point in tandem.

Collect data

end

\section{Simulation Results}

We have evaluated the performance of ORP-MS with existing algorithm DECA [22], EEHC [32] and ES-WCA [27] by coding using MATLAB 2017a.The experimental scenario parameters are listed in Table II.

Performance comparison of ORP-MS and existing algorithms are as follows:

\section{A. Number of Active Sensors and Ative Cluster Heads}

Fig. 9 and 10 show number of active cluster heads and number of active nodes per round respectively for entire simulation duration. Fig. 9 reflects the number of active $\mathrm{CHs}$ per round. Due to static sink approach and single hop communication $\mathrm{CH}$ in EEHC drain energy quickly. However, DECA, ES-WCA and ORP-MS show improvement due to mobile sink approach. Furthermore, as the isolated nodes are also being clustered and rendezvous point is selected for every round ORP- MS shows bodacious improvement over previous schemes.

Fig. 10 shows the number of active sensors per round. It is clear that in DECA and ORP-MS all the sensors are active since starting owing to the fact that both the schemes bind unconnected sensors as well. Further, we see considerable improvement in ORP-MS scheme, as all the sensors are active for more than around $1200^{\text {th }}$ round. Fig. 10 clearly indicates that the more nodes are alive for longer number of rounds that means network life is prolonged using ORP-MS approach. 


\section{B. Remaining Energy}

Fig. 11 shows the average remaining energy of entire network. This graph has a gradual downwards slope indicating uniform energy consumption in the network which further depicts the efficient and well-balanced consummation of Proposed ORP-MS algorithm.

Table 2: Network parameters

\begin{tabular}{|l|l|}
\hline Radius of Target area & $50 \mathrm{~m}$ \\
\hline No. of sensors & 100 \\
\hline No. of CHs & $15-30$ \\
\hline Initial energy of sensors & $.02 \mathrm{~J}$ \\
\hline Initial energy of sensors & $.1 \mathrm{~J}$ \\
\hline $\mathrm{T}^{\mathrm{x}}$ range of sensors & $10 \mathrm{~m}$ \\
\hline Size of Control packet & $100 \mathrm{bits}$ \\
\hline Size of Data packet & $500 \mathrm{bits}$ \\
\hline Multi-path fading & $.0013 \mathrm{pJ} / \mathrm{bit} / \mathrm{m} 4$ \\
\hline $\mathrm{T}^{\mathrm{x}}$ or $\mathrm{R}^{\mathrm{x}}$ electronics & $50 \mathrm{~nJ} / \mathrm{m} 2 / \mathrm{bit}$ \\
\hline
\end{tabular}

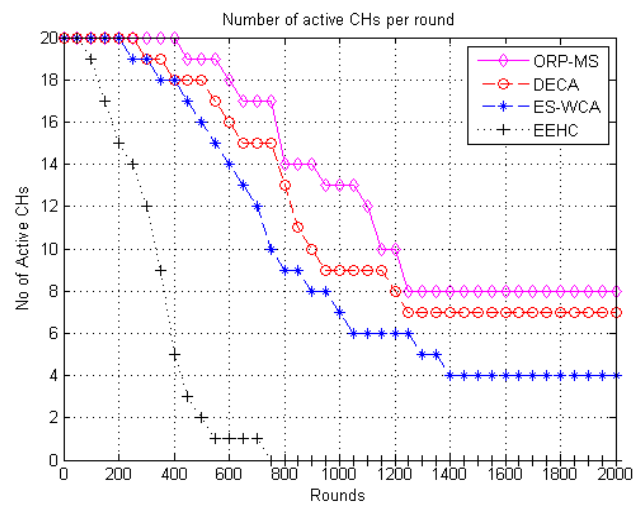

Fig. 9: Number of active $\mathrm{CH}$ per round

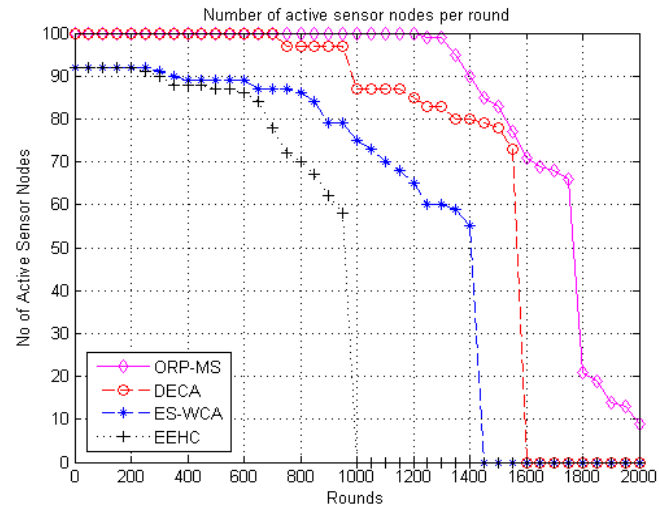

Fig. 10: Number of active nodes per round

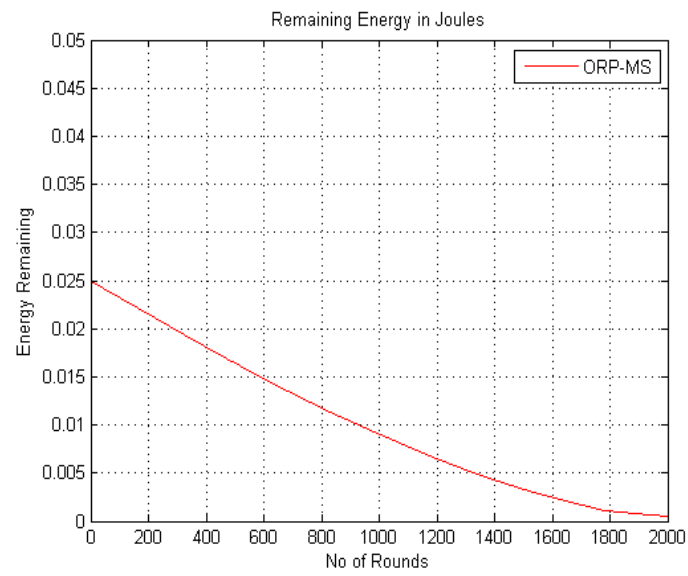

Fig. 11: Remaining energy in Joules

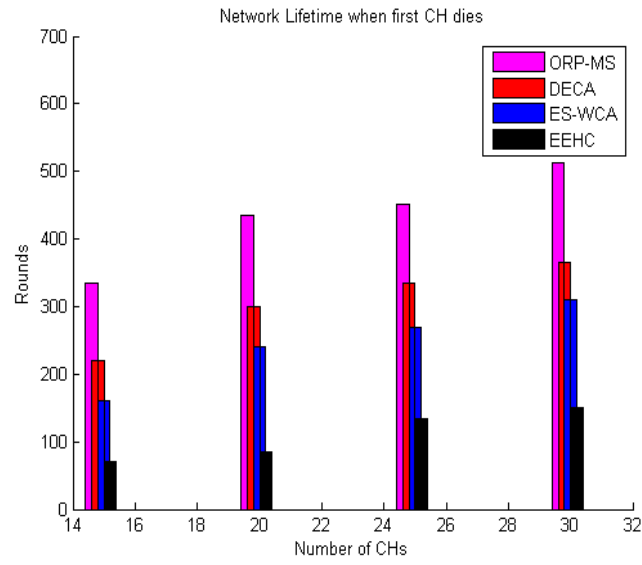

Fig. 12: Network lifetime

\section{Network lifetime}

In Fig. 12, network lifetime is evaluated of the WSNs by Changing the number of zones thereby changing number $\mathrm{CHs}$ in the network from 15-30. The benchmark of lifetime is assigned on the round at which the first $\mathrm{CH}$ dies. From Fig. 12, we can observe that ORP-MS has outperformed the existing algorithms in terms of networks lifetime.

\section{Throughput}

Fig. 13 depicts deployed scenario. It is bodacious that as the network reaches around $1800^{\text {th }}$ round, only 20 nodes are found to be alive in the network. This means that further increase in the throughput would be less gradual as equated with the earlier rounds. And after the $2000^{\text {th }}$ round, the value of throughput becomes constant indicating that nodes have practically died out and no further communication is happening in the network.

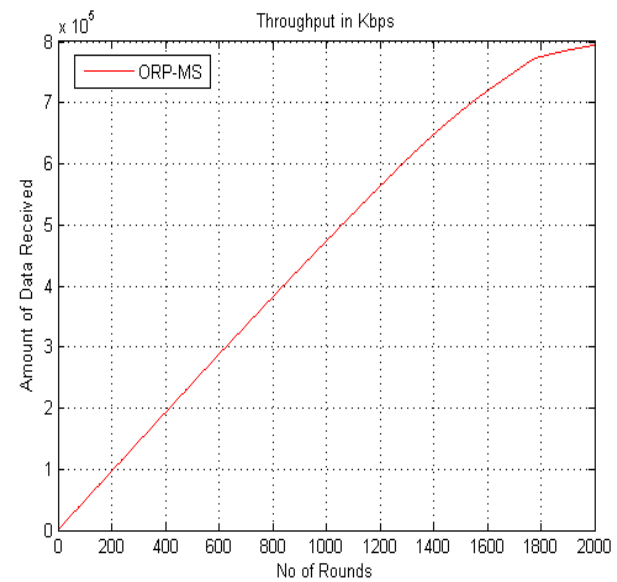

Fig. 13: Throughput (summation of subsequent rounds)

\section{Conclusion}

In this work, a new clustering algorithm using mobile sink with varying rendezvous point is proposed named Optimal Rendezvous Point Selection using Mobile Sink (ORP-MS). The proposed algorithm makes sure minimum isolated nodes and variable rendezvous point that minimum energy is drained in transmitting information to the sink. For every round rendezvous point is selected such that it approximately lies in mid of $\mathrm{CH}, \mathrm{CH}$ of isolated nodes and leftover node if any, as shown in Fig. 7. The simulation result proves that ORP-MS has an edge over other existing schemes; viz. DECA, EEHC and ES-WCA. The substantial improvement was seen terms of network lifetime, energy efficiency and high throughput is achieved. 


\section{References}

[1] M. Krysander and E. Frisk, "Sensor Placement for Fault Diagnosis," IEEE Trans. Syst. Man Cybern. Part A Syst. Humans, vol. 38, no. 6, pp. 1398-1410, 2008.

[2] S. Zhang and H. Zhang, "A Review of Wireless Sensor Networks and Its Applications," Proceeding IEEE Int. Conf. Autom. Logist., no. August, pp. 386-389, 2012.

[3] V. Vimal and M. J. Nigam, "Ensuring Uniform Energy Consumption in Non- Deterministic Wireless Sensor Network to Protract Networks Lifetime," Int. J. Electron. Commun. Eng., vol. 11, no. 9, pp. 966-970, 2017.

[4] M. A. Matin and M. M. Islam, Overview of Wireless Sensor Network. InTech, 2012.

[5] Z. Iqbal and H.-N. Lee, "Deployment Strategy Analysis for Underwater Cooperative Wireless Sensor Networks," 2015 Int. Conf. Inf. Commun. Technol. Converg., pp. 699-703, 2015.

[6] L. B. Bhajantri and N. Nalini, "Cluster Based Optimization of Routing in Distributed Sensor Networks Using Bayesian Networks with Tabu Search," vol. 60, no. 2, pp. 199-208, 2014

[7] W. R. Heinzelman, A. Chandrakasan, and H. Balakrishnan, "Energy-Efficient Communication Protocol for Wireless Microsensor Networks," Proc. 33rd Annu. Hawaii Int. Conf. Syst. Sci., vol. 0, no. c, pp. 3005-3014, 2000.

[8] L. Qing, Q. Zhu, and M. Wang, "Design of a distributed energyefficient clustering algorithm for heterogeneous wireless sensor networks," Comput. Commun., vol. 29, no. 12, pp. 2230-2237, 2006.

[9] L. Zhao and Q. Liang, "Medium-Contention Based EnergyEfficient Distributed Clustering (MEDIC) for Wireless Sensor Networks," Int. J. Distrib. Sens. Networks, vol. 3, no. 4, pp. 347369, 2007.

[10] C. H. Lin and M. J. Tsai, "A comment on 'HEED: A Hybrid, Energy-Efficient, Distributed clustering approach for ad hoc sensor networks,"' IEEE Trans. Mob. Comput., vol. 5, no. 10, pp. 14711472, 2006.

[11] T. Chiang, "Regional Energy Aware Clustering with Isolated Nodes in Wireless Sensor Networks," pp. 1829-1833, 2014

[12] B. Li, H. Li, W. Wang, Z. Hu, and Q. Yin, "Energy-Effective Relay Selection by Utilizing Spacial Diversity For Random Wireless Sensor Networks," IEEE Commun. Lett., vol. 17, no. 10, pp. 1972 1975, 2013.

[13] H. Asharioun, H. Asadollahi, T. C. Wan, and N. Gharaei, "A Survey on Analytical Modeling and Mitigation Techniques for the Energy Hole Problem in Corona-Based Wireless Sensor Network," Wirel. Pers. Commun., vol. 81, no. 1, pp. 161-187, 2015

[14] M. T. Ha, T. D. Le, and H. Choo, "Employing a Novel Two Tiered Network Structure to Extend the Lifetime of WSNs," IEEE Wirel. Commun. Netw. Conf. WCNC, 2009.

[15] C. Tunca, S. Isik, M. Y. Donmez, and C. Ersoy, "Distributed Mobile Sink Routing for Wireless Sensor Networks: A Survey," IEEE Commun. Surv. Tutorials, vol. 16, no. 2, pp. 877-897, 2014.

[16] W. Liang, J. Luo, and X. Xu, "Prolonging Network Lifetime via A Controlled Mobile Sink in Wireless Sensor Networks," GLOBECOM - IEEE Glob. Telecommun. Conf., 2010.

[17] M. Di Francesco, S. K. Das, and G. Anastasi, "Data Collection in Wireless Sensor Networks with Mobile Elements," ACM Trans. Sens. Networks, vol. 8, no. 1, pp. 1-31, 2011

[18] V. Kaundal, P. Sharma, and M. Prateek, "Wireless Sensor Node Localization based on LNSM and Hybrid TLBO- Unilateral technique for Outdoor Location," Int. J. Electron. Telecommun., vol. 63, no. 4, pp. 389-397, 2017.

[19] M. J. Handy, M. Haase, and D. Timmermann, "Low Energy Adaptive Clustering Hierarchy with Deterministic Cluster-Head Selection," 2002 4th Int. Work. Mob. Wirel. Commun. Network, MWCN 2002, pp. 368-372, 2002.

[20] H. Salarian, K.-W. Chin, and F. Naghdy, “An Energy Efficient Mobile Sink Path Selection Strategy for Wireless Sensor Networks," IEEE Trans. Veh. Technol., vol. 63, no. c, pp. 1-1, 2014.

[21] A. Kaswan, K. Nitesh, and P. K. Jana, "Energy Efficient Path Selection for Mobile Sink and Data Gathering in Wireless Sensor Networks," AEU - Int. J. Electron. Commun., vol. 73, pp. 110-118, 2017.
[22] N. Mazumdar and H. Om, "Distributed energy-efficient clustering algorithm for mobile-sink based wireless sensor networks," Proc. 10th Int. Conf. Intell. Syst. Control. ISCO 2016, 2016

[23] P. Chanak, I. Banerjee, J. Wang, and R. Sherratt, "Obstacle Avoidance Routing Scheme through Optimal Sink Movement for Home Monitoring and Mobile Robotic Consumer Devices," IEEE Trans. Consum. Electron., vol. 60, no. 4, pp. 596-604, 2014.

[24] A. W. Khan, A. H. Abdullah, M. A. Razzaque, and J. I. Bangash, "VGDRA: A Virtual Grid based Dynamic Routes Adjustment Scheme for Mobile Sink based Wireless Sensor Networks," IEEE Sens. J., vol. 15, no. 1, pp. 1-7, 2014.

[25] O. Cayirpunar, E. Kadioglu-Urtis, and B. Tavli, "Optimal Base Station Mobility Patterns for Wireless Sensor Network Lifetime Maximization," IEEE Sens. J., vol. 15, no. 11, pp. 6592-6603, 2015.

[26] F. Tashtarian, M. H. Yaghmaee Moghaddam, K. Sohraby, and S. Effati, "On Maximizing the Lifetime of Wireless Sensor Networks in Event-Driven Applications With Mobile Sinks," IEEE Trans. Veh. Technol., vol. 64, no. 7, pp. 3177-3189, 2015.

[27] D. Amine, B. Nasr-Eddine, and L. Abdelhamid, "A Distributed and Safe Weighted Clustering Algorithm for Mobile Wireless Sensor Networks," Procedia Comput. Sci., vol. 52, no. 1, pp. 641-646 2015.

[28] M. Z. Hasan, H. Al-Rizzo, and M. Günay, "Lifetime Maximization by Partitioning Approach in Wireless Sensor Networks,” Eurasip J. Wirel. Commun. Netw., vol. 2017, no. 1, pp. 1-18, 2017.

[29] L. Xie, Y. Shi, Y. T. Hou, W. Lou, H. D. Sherali, and S. F. Midkiff, "Multi-node wireless energy charging in sensor networks," IEEE/ACM Trans. Netw., vol. 23, no. 2, pp. 437-450, 2015.

[30] R. Prasad, P. Mathur, R. H. Nielsen, and N. R. Prasad, "Data Collection Using Miniature Aerial Vehicles in Wireless Sensor Networks," IET Wirel. Sens. Syst., vol. 6, no. 1, pp. 17-25, 2016.

[31] P. Bose and M. Gurusamy, "Bacteria Foraging Algorithm Based Optimal Multi Sink Placement in Wireless Sensor Networks," J. Intell. Syst., vol. 0, no. 0, 2017.

[32] D. Kumar, T. C. Aseri, and R. B. Patel, "EEHC: Energy Efficient Heterogeneous Clustered Scheme for Wireless Sensor Networks,' Comput. Commun., vol. 32, no. 4, pp. 662-667, 2009. 